\title{
The Operation and Model of UPQC in Voltage Sag Mitigation Using EMTP by Direct Method
}

\author{
S. Mehdi Hosseini ${ }^{a^{*}}$ \\ ${ }^{a}$ Department of Electrical, South Tehran science and Research Branch, Islamic Azad University, Tehran, Iran
}

\begin{abstract}
Nowdays, regarding to the great us of sensitive devices, the quality of the transmitted power, have become an important issue. One of the most important dimension of power quality is magnitude load terminal voltage. Different devices has been designed for voltage sag compensation or other distortions. UPQC is a compensator which is able to compensate all distortions, such as voltage sag, flicker, unbalance, harmonic and current distortions such as reactive, harmonic and unbalance. Several controllers have been designed to compensate the whole or a part of distortions. In this paper its going to introduce a kind of controller which is able to compensate voltage sag to direct and simple by UPQC. At the end, simulation and voltage sag mitigation is going to be performed by ATP-EMTP software.
\end{abstract}

Keywords:

UPQC;

Voltage sag;

Compensator;

EMTP;

Hysteresis.

Article History:

Received: 28 January 2018

Accepted: 16 May 2018

\section{1- Introduction}

There are lots of industrial consumers which are sensitive to voltage drop. Voltage drop may occur in various duty time some of these duty cycle are so small but harmful for some device's functions. So it's necessary to design a device which is able to compensate all distortions very fast. Almost $85 \%$ of power quality problems refer to voltage drop. Voltage sag means RMS magnitude drop lower than $90 \%$ of the nominal voltage. There may be lots of reasons for voltage sag. One of them is fault in the load connected to the feeder, in the other words Pcc point or induction motor starting up which needs high current at the starting time. Voltage drop of the supply or the network is the other reason $[1,2]$.

The UPQC compensator consists of a DVR and a D-statcom in which a capacitor is placed instead of DC supply and these two are connected from that point. It can be expected parallel compensator in other words DStatcom to compensate, current distortions and to transmit power into the dc capacitor and to charge it. If an independent voltage supply is not connected to the network, current distortion will affect supply productive voltage. Therefore if current distortion could be compensated, we can come over most of voltage problems [3-6].

In comparison to DVR, UPQC can accomplish the aims of DVR usage meanwhile it does not need independence dc supply. Also, according to what have been mentioned, because of current distortion elimination before their entrance into series transformer (COPEL), their effectiveness will be higher [7-10].

Voltage problems which distribution lines encounter are:

1. Voltage sag/swell; 2. Unbalance; 3. Distorted wave forms due to harmonics.

Voltage sag/swell causes because of fault occurrence in feeder or voltage supply itself.

Unbalance may cause because of unbalanced load current and the effectiveness of the supply. Moreover it should be mentioned that there are two kinds of unbalance:

\footnotetext{
* CONTACT: nemehdi@yahoo.com

DOI: http://dx.doi.org/10.28991/esj-2018-01138

(C) This is an open access article under the CC-BY license (https://creativecommons.org/licenses/by/4.0/).
} 
a. Magnitude; b. phase angle

When fault is three phase, the unbalance will be in the form of magnitude. When the fault is phase to ground or two phase fault will be both in magnitude and phase when unbalance occurrence is because of the supply source fault, it can be in any form.

When sensitive look is single phase, so unbalance on phase would not be important. Only when the fault is single phase to ground or two phase and sensitive load is also three phase, so a synchronizer like PLL (phase locked loop) with a compensator will be needed lots of controlling methods have been designed to control UPQC which are mostly base on one or some changes (transformations). The applied transformations usually are park transformation fortescue transformation (ido) and Clark transformation (ab). Each of these transformation has special characteristic and could be applied for a special purpose [11].

The methods which are based on park transformation are used for separation of harmonic distortions and reactive current from the main wave. The problem is that when park transformation is used, it's needed to specify the positive sequence phase (without zero and negative sequences) of use PLL in each condition, even the complete symmetry.

In park transformation harmonic and reactive distortions are possible to separate so, the direction of parallel compensator, and reactive current elimination and harmonic of the load should mostly considered. It should be mentioned that in park transformation the frequency of the transformation should be consider to the same frequency as the network frequency [12-15].

\section{1-1- Calculation of Reference Signal}

Use Park Converter (dq0) in parallel converter. Consider the following Figure 1:

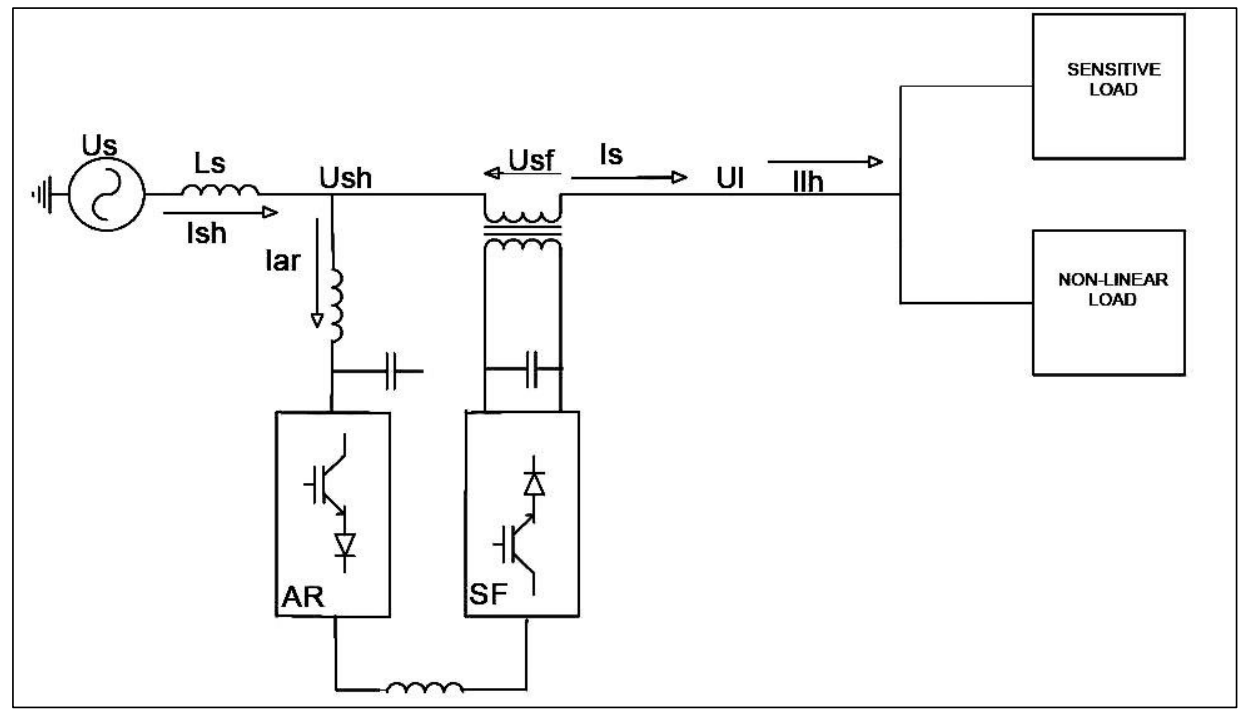

Figure 1. Three-phase current transformers in UPQC topology.

The series filter (SF) is designed to correct harmonics of voltage supply, voltage drop and voltage rise, and unbalanced voltage. The control equation is as follows:

$$
u_{s f}=u_{c o m p}
$$

$U_{\text {comp, }}$ The load voltage compensator is required to eliminate voltage supply changes, which consists of two parts, one based on the dq theory to detect harmonics and reactive current, and the other is derived from the transformation of Foreski for non-equilibrium correction. The active rectifier (AR) is used to control the actual power transmission (to or from) the DC bus and also to control the current DC bus bay for the purpose of having a single power factor, while filtering the current harmonics and reactive power amplifier AR Shunt). The DC Boss current is controlled by different controllers. The rectifier control equation (shunt converter) is as follows:

$$
I_{a r}=I_{a r 1}+I_{p f}
$$

$\mathrm{I}_{\mathrm{ar} 1}$ is the first harmonic of the current in the rectifier input created by the dc flow control loop and $\mathrm{I}_{\mathrm{pf}}$ is the current component for the harmonics to be filtered and the power factor correction 
The control methods used for parallel and series active filters typically use instantaneous reactive power theory to calculate reference signals. This theory offers a powerful tool, but its implementation is difficult in practice because it requires a large number of analogue mixers, splitters, filters, and so on. Advances in $D_{\text {sp }}$ technology reduce the speed of computing with the rapid conversion of $\mathrm{A} / \mathrm{D}$ and hardware to various applications and reduce the hardware requirement soft circuit. The dq components of each of the voltages and currents shown in Fig. 4 are obtained from the equations below [5]:

$$
\left[\begin{array}{l}
u_{o} \\
u_{d} \\
u_{q}
\end{array}\right]=\sqrt{\frac{2}{3}} T \cdot\left[\begin{array}{l}
u_{a} \\
u_{b} \\
u_{c}
\end{array}\right] \quad \text { and } \quad\left[\begin{array}{l}
i_{o} \\
i_{d} \\
i_{q}
\end{array}\right]=\sqrt{\frac{2}{3}} T \cdot\left[\begin{array}{l}
i_{a} \\
i_{b} \\
i_{c}
\end{array}\right]
$$

The transformation matrix $\mathrm{T}$ stands for:

$$
\theta=\theta_{0}+\int_{0}^{t} \operatorname{\omega td} t \quad \text { and } \quad T=\left[\begin{array}{ccc}
\frac{1}{\sqrt{2}} & \frac{1}{\sqrt{2}} & \frac{1}{\sqrt{2}} \\
\operatorname{Cos} \theta & \operatorname{Cos}\left(\theta-\frac{2 \pi}{3}\right) & \operatorname{Cos}\left(\theta-\frac{4 \pi}{3}\right) \\
-\operatorname{Sin} \theta & -\operatorname{Sin}\left(\theta-\frac{2 \pi}{3}\right) & -\operatorname{Sin}\left(\theta-\frac{4 \pi}{3}\right)
\end{array}\right]=T^{\text {Trans } p}
$$

$\theta$ the initial angle of the voltage is described in detail before. $\theta_{0}$ the same angle that was obtained in PLL. And the currents can be decomposed into two DCs (Hz50 main frequency) and AC (convergent and transonic) to the dq0 device (containing harmonics and inter Harmonic). Thus, the relationships are as follows:

$$
i_{d}=\bar{i}_{d}+\tilde{i}_{d} \quad, \quad i_{q}=\bar{i}_{q}+\tilde{i}_{q}
$$

$I_{d}$ is a reactive component and $i_{q}$ is the active power component. The extracted AC and DC components are as follows:

$$
\begin{aligned}
& \tilde{i}_{d}(z)=H P F(z) i_{d}(z), \quad \tilde{i}_{q}(z)=\operatorname{HPF}(z) i_{q}(z) \\
& \bar{i}_{d}(z)=i_{d}(z)-\tilde{i}_{d}(z) \quad, \quad \bar{i}_{q}(z)=i_{q}(z)-\tilde{i}_{q}(z) \\
& H P F_{(z)}=H P F_{(s)}\left|s=\frac{2}{T} \frac{1-z^{-1}}{1+z^{-1}}=\frac{s}{s+\omega_{c}}\right| s=\frac{2}{T} \frac{1-z^{-1}}{1+z^{-1}}=\frac{2\left(1-z^{-1}\right)}{\left(2+\omega_{c} T\right)-\left(2-\omega_{c} T\right) z^{-r}}
\end{aligned}
$$

$\mathrm{T}$ is time of sampling time. The sampling time for the proper filtering is minimal $T<T_{h} / 4, T_{h}$ which is the period of rotation, the largest harmonic to be eliminated $T_{h}=1 / f$. Therefore, switching is based on the required power and switching frequency. In the case of the removal of some low-frequency and high-frequency harmonics, we can use switches with lower switching frequencies and cheaper ones. For example, if the largest harmonic is 21 (the harmonic whose compensation is important to us) then $\mathrm{T}$ It will be almost a millisecond.

Accordingly, for the removal of harmonics, the reference currents for the parallel AR filter are calculated from formula 9-1. If we want to correct the power factor with the elimination of the harmonics (reactive power compensation), then the reference currents are calculated from Equation 10.

$$
\left[\begin{array}{c}
i_{p f a}^{*} \\
i_{p f b}^{*} \\
i_{p f c}^{*}
\end{array}\right]=\sqrt{\frac{2}{3}}\left[\begin{array}{cc}
\operatorname{Cos} \theta & -\operatorname{Sin} \theta \\
\operatorname{Cos}\left(\theta-\frac{2 \pi}{3}\right) & -\operatorname{Sin}\left(\theta-\frac{2 \pi}{3}\right) \\
\operatorname{Cos}\left(\theta+\frac{2 \pi}{3}\right) & -\operatorname{Sin}\left(\theta+\frac{2 \pi}{3}\right)
\end{array}\right]\left[\begin{array}{c}
\tilde{i}_{l h d} \\
\tilde{i}_{l h q}
\end{array}\right]
$$




$$
\left[\begin{array}{c}
i_{p f a}^{*} \\
i_{p f b}^{*} \\
i_{p f c}^{*}
\end{array}\right]=\sqrt{\frac{2}{3}}\left[\begin{array}{cc}
\operatorname{Cos} \theta & -\operatorname{Sin} \theta \\
\operatorname{Cos}\left(\theta-\frac{2 \pi}{3}\right) & -\operatorname{Sin}\left(\theta-\frac{2 \pi}{3}\right) \\
\operatorname{Cos}\left(\theta+\frac{2 \pi}{3}\right) & -\operatorname{Sin}\left(\theta+\frac{2 \pi}{3}\right)
\end{array}\right]\left[\begin{array}{l}
i_{l l d}+\tilde{i}_{l h d} \\
\tilde{i}_{l h q}
\end{array}\right]
$$

The reference voltages for the active-series filter (serial converter) are exactly the same as the reference current for parallel filters. Thus, if only harmonic correction is required, reference voltages are obtained from (11), but if, in addition to the correction of the harmonics, we want to correct the voltage size, then the reference voltage of the Equations 12 and 13 are obtained.

$$
\begin{aligned}
& {\left[\begin{array}{l}
u_{s f a}^{*} \\
u_{s f b}^{*} \\
u_{s f c}^{*}
\end{array}\right]=\sqrt{\frac{2}{3}} T^{-1}\left[\begin{array}{l}
\tilde{u}_{s h d} \\
\tilde{u}_{s h q} \\
u_{s h o}
\end{array}\right]} \\
& {\left[\begin{array}{l}
u_{s f a}^{*} \\
u_{s f b}^{*} \\
u_{s f c}^{*}
\end{array}\right]=\sqrt{\frac{2}{3}} T^{-1}\left[\begin{array}{l}
\tilde{u}_{s h d} \\
\tilde{u}_{s h q} \\
u_{s h o}
\end{array}\right]+\left[\begin{array}{l}
u_{c o m p a} \\
u_{c o m p b} \\
u_{\text {compc }}
\end{array}\right]}
\end{aligned}
$$

That is, the voltage compensation factor is:

$$
\left[\begin{array}{l}
u_{\text {coma }} \\
u_{\text {comb }} \\
u_{\text {comc }}
\end{array}\right]=\sqrt{\frac{2}{3}} T^{-1}\left[\begin{array}{l}
u_{\text {comd }} \\
u_{\text {comq }} \\
u_{\text {como }}
\end{array}\right] \text { and }\left[\begin{array}{l}
u_{\text {compd }}(s) \\
u_{\text {compq }}(s) \\
u_{\text {compo }}(s)
\end{array}\right]=\left[\begin{array}{l}
u_{\text {dnom }}(s)-u_{d}(s) \\
u_{\text {qnom }}(s)-u_{q}(s) \\
u_{\text {onom }}(s)-u_{o}(s)
\end{array}\right]
$$

Given that the unbalanced compensation method for voltage is based on the elimination of unbalanced elements. After the conversion of the three phases to the optional reference system and the separation of the active and reactive elements, or the positive sequence and the negative sequence, the reactive element and zero, or the negative and zero sequences, are set to zero and the main element is reversed.

Then measured values has been reduced and converted the result that the residual unbalanced or reactive elements with a phase difference of 180 degrees are reversed. But here again the harmonics production method is used because the reason for the creation of voltage harmonics is the current harmonics.

In methods in which fortescue transformation is used, the aim is unbalance elimination or the correct function in unbalance situations. The unbalance, here means the phase unbalance. All the mentioned transformation are used for getting a suitable reference. The comparison and then the production of semiconductor fire signal will be done, after a reference was made. So, when the voltage is unbalance (phase), we can't accept the network voltage or supply as the reference. So, it is necessary to use fortescue transformation. The Fortescue transformation needs instantaneous angle specification of each phase. So, for using this transformation, phase detector of phase estimation should be used.

One important point to be mentioned here is that accessing a perfect and correct reference is also possible without Fortescue transformation. In this method the phase angle and the positive sequential main frequency of each phase is specified by PLL, then by a signal generator, sine wave is produced and multiplied by the suitable gain. So we can access the instantaneous quantity of the positive sequence and the main frequency of each phase.

Note that the load current distortion should be produced and the source can't supply the load need of distortion but it should be supplied by compensator and in compensation of voltage distortion, the distortion elimination should be the aim.

As you can see, the volume of calculations is very high with the above methods. A new method of control and the produce fire signal has been introduced by the authors. Then the voltage sag is going to be considered by EMTP software and the simulation results is going to be represented.

\section{2- UPQC Structure}

As it mentioned UPQC consist of a DVR compensator and a D-Statcom and it partially has the both compensator's advantages. Besides its compensatory advantage in both parallel and series (parallel filter and series filter) it has another advantage, which is using common dc source and it can be changed by UPQC itself. UPQC transfers power from one 
part to another. The power used by series compensator for voltage compensation is provided by a parallel compensator from the system. So, if UPQC be assumed lossless, the power of the source or network will be fixed in the situation of fault occurrence. While the voltage of the source drops, injected current from source increases. Notice UPQC in this figure:

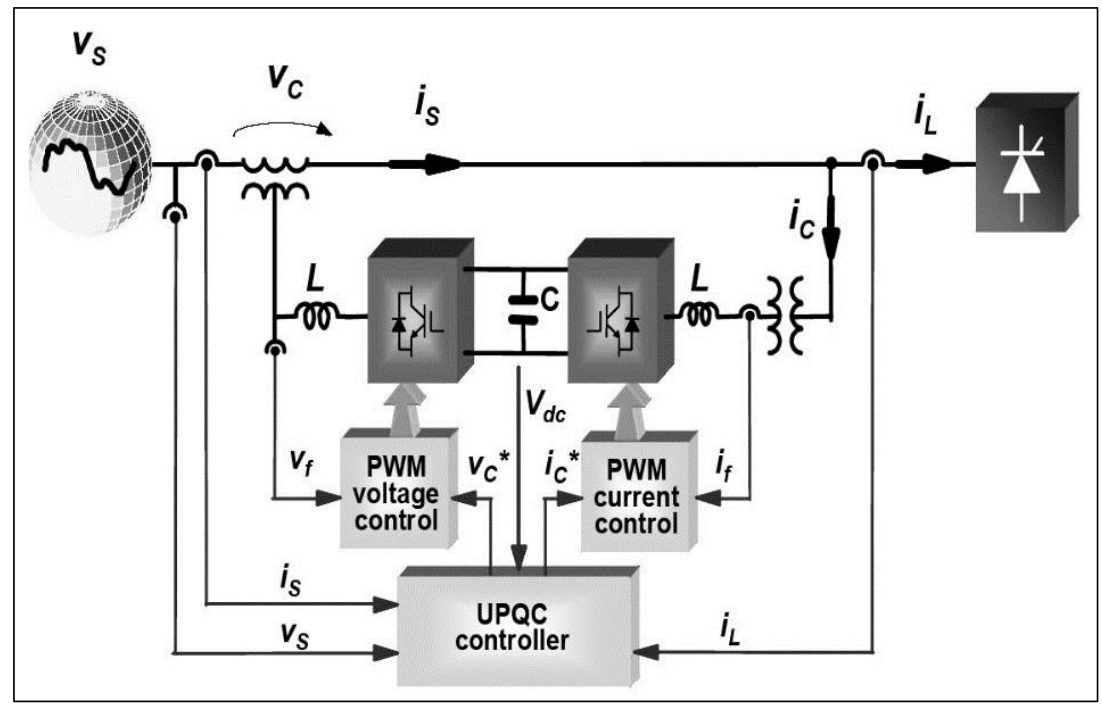

Figure 2. Structure of UPQC (Unified Power Quality Condition) [4].

Parallel compensator can be placed on any sided of series compensator and when the load is too distorted, the parallel compensator is usually placed near the load to prevent current distortion's entry toward voltage source series compensator. In the case of remaining current distortion even after parallel compensator, self of series transformer (couple).

\section{3- The Control Method}

In this method no transformation is used for voltage sag compensation and all distortion as harmonics is regarded as magnitude and compensates in company with voltage drop. The reason is that the compensator is done every moment not in a period.

The strategy is, after voltage instantaneous magnitude and reference magnitude comparison. The error signal enters Hysteresis band. This band produce signals, when error signal contains positive and extremely high hysteresis it produces fire signal for the higher and the positive switch and when error signal contains negative and less than lower limit magnitude, it produces fire signal for the lower and the negative pole. So this controller is able to distinguish and compensate all kinds of voltage sag which caused by fault occurrence the change in wave shape and the voltage drop caused by harmonics. This controller's structure is as follow:

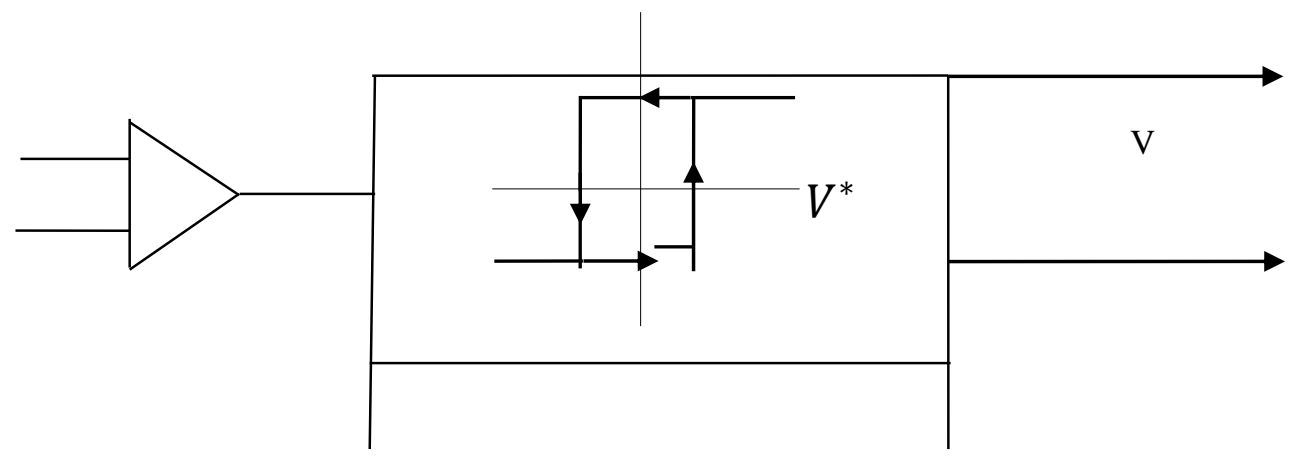

Upper switch

Figure 3. Hysteresis loop-generating 2-level signals

This is another compensatory method. It's difference from this method is that an integrator and a comparator is used in place of the hysteresis band. The integrator causes delay in system and doesn't cover some fast transients. For parallel compensator controlling Fortescue transformation is used to separate distortions. Parallel compensator not only compensates current distortions but is also, supplied the required power for series compensator. The control loop of required power for series compensator by parallel compensator is as follow: 
APL controller is used in this loop which controls capacitor change due to the source limitation in productive current and attributing voltage error magnitudes of dc capacitor to the current magnitude and the need of organizing control loop of DC capacitor voltage has removed.

\section{4- Simulation and Results}

Electro Magnetic Transient Program (ATP-EMTP) is a well know simulator among power engineers, for its reliability, effectiveness and coincidence with practice. In this section we use this simulator to examine the performance of the UPQC controlled by the mentioned algorithm, in mitigation voltage sag side effects on load.

Among possible voltage sag conditions, it has been chosen single line to ground fault on the parallel feeder. One of the most common causes of voltage sag is the single line to ground fault on the adjacent feeder, as shown in Fig.4.

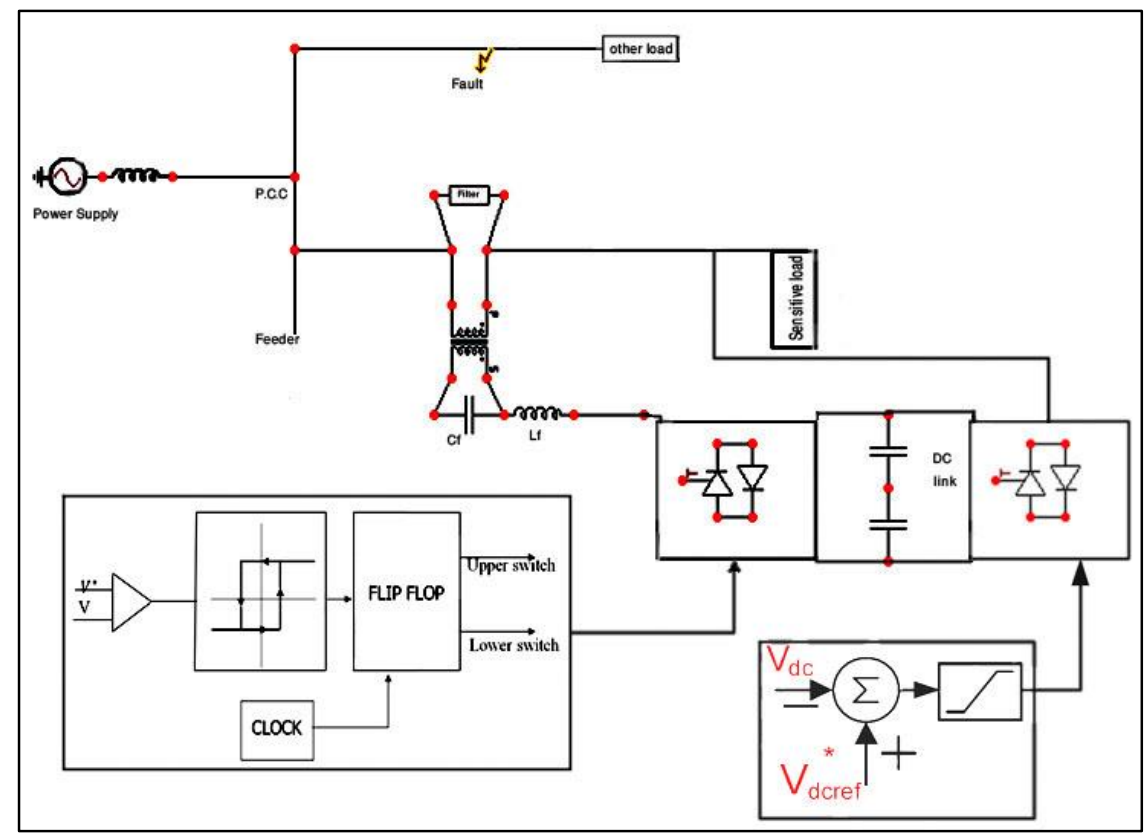

Figure 4. UPQC simulation model

In the event of a connection in the normal load split, the voltage drop will occur in the P.C.C waveguide. The sensitive time will also be affected by the drop in its terminal. Therefore, the compensation function at this time will compensate for this voltage drop. We assume that the mains supply is the network and its voltage is symmetric. Also, the voltage of the source itself does not become asymmetric. Alternatively, we can use PLL to determine the fundamental frequency angle of the positive sequence at the moment of the phase a measurement. Given the assumption of the voltage symmetry the source and the proposed method are used to compensate for the single-phase model. Therefore, the coupling is considered to be a single-phase connection to the earth. Also, ground resistance is considered zero-ohm for determining the highest voltage drop at P.C.C point. The connection time is $83 \mathrm{~ms}$ and the duration is $42 \mathrm{~ms}$.

\section{4-1- Circuit Specifications}

$$
\begin{array}{ll}
C f=20 \mu F & \text { RCfilter: } 1 \mathrm{ohm}, 20 \mu F \\
L f: 1 \mathrm{mH} & \mathrm{Z} 1=\mathrm{Z} 2=\mathrm{Z} 3=2.5+\mathrm{j} 2 \mathrm{ohm}
\end{array}
$$

Vdc: v $2 \times 75 \quad$ Vsource: $\sin (w t) 220 \sqrt{2}$

\section{4-2- Simulation Results}

In the case where the other phase feeder is connected to the ground (zero resistance), we test the circuit from 0.08 to 0.125 seconds. It is assumed that the voltage decreases at this point in the pcc point, and this decreases to the sensitive load, ie, the other branch of the feeder. The simulation results using the ATP-EMTP software have proven that we have managed to quickly achieve a simple, cheap, fast and easy control, and we also recommend using this type of control. 


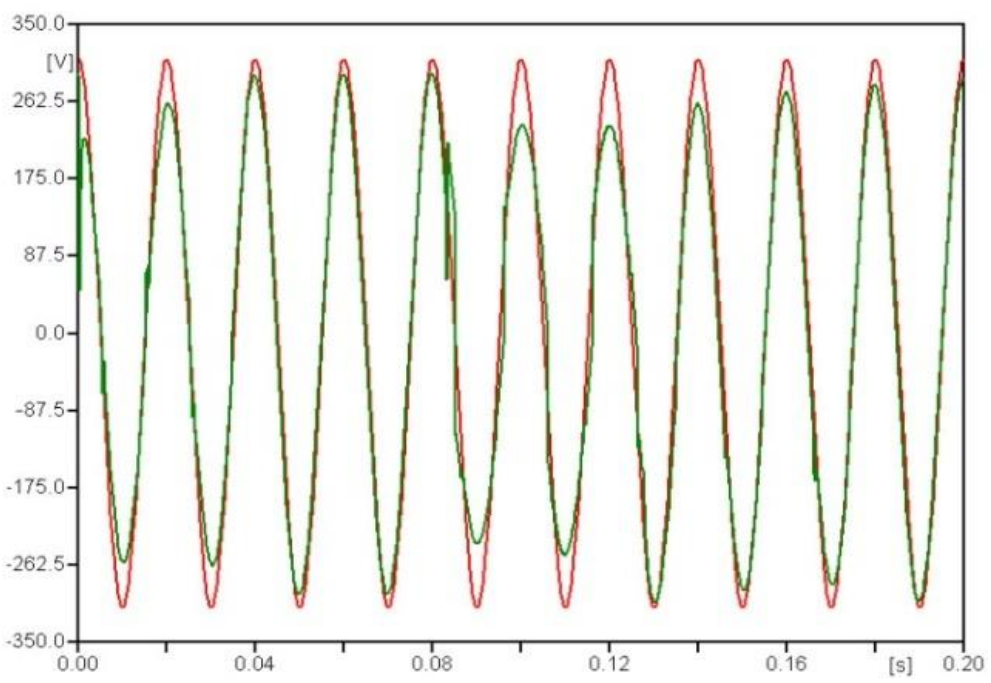

Figure 5. Terminal voltage at fault time with UPQC compensation

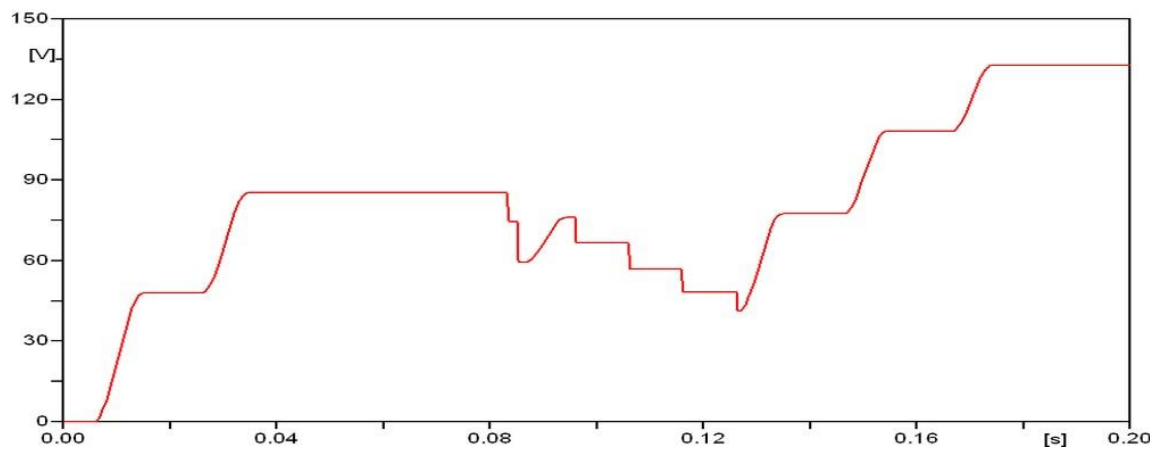

Figure 6. Voltage of dc capacitors at voltage sag

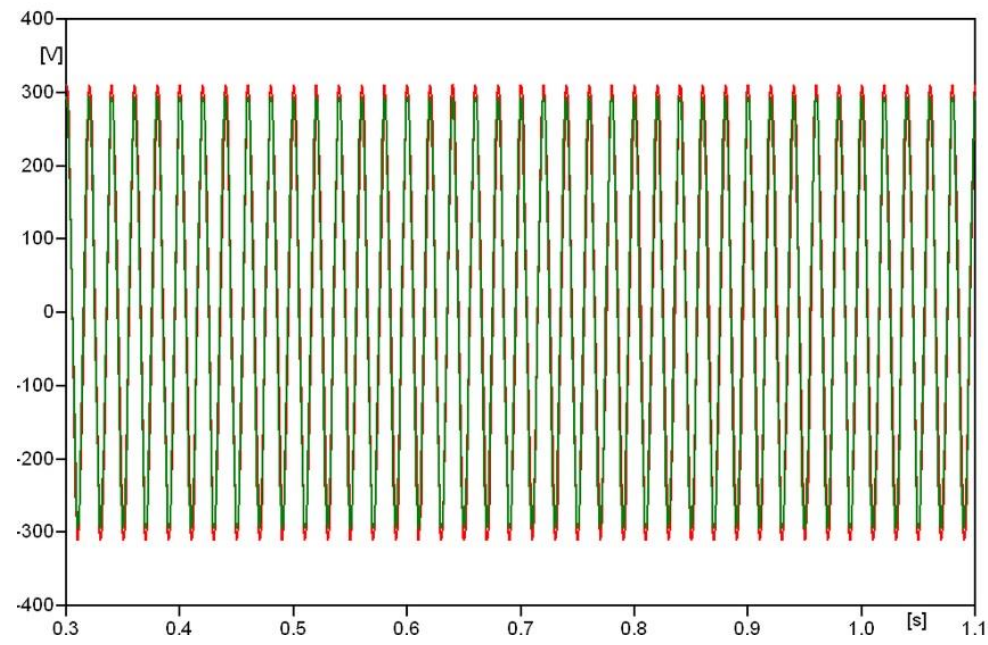

Figure 7. Investigate the voltage in the steady state with the voltage sag

As you can see, this method does not require any processor, and the pi controller is also not used, so almost no delay can be compensated and the fastest compensation is taken into account. All transitions can also be compensated for because there are no delayed elements in its control. Its operation is very similar to the transducer's spark arresters or lightning strings, which, when the excess voltage exceeds a defined value, is discharged to the ground. Here, the hysteresis loop performs a similar operation, so that if the error signal is of a certain value More or less the switch is needed to compensate for the voltage. Other compensations can also be performed at the same time, such as compensating voltage harmonics.

In this method, due to the instantaneous compensation, there is no need for large and complex computations such as those described in this paper, nor does it require a specific controller. At the same time, compensation of the voltage drop of any other turbulence in the waveform can be as a voltage drop or amplification And a momentary compensation 
such as a voltage drop or a voltage surge In order to eliminate transient disturbances caused by switching, it is possible to use passive filters. Of course, optimal selection of link capacitor capacities is also very important. But in the previous methods, they have often used a very large amount of computational method.

All of the goals are relative and are not recommended for use at all, and only for better explanation of the reasons for using the conversions. In this research, we use the reactive power and current harmonics to compensate for the shunt's inverters, and we have to use dqo conversion, but we can eliminate current disturbances by passive filters to a reasonable degree. Because the passive filters have been widely used in the industry, they have been able to completely eliminate reactive power with regulators and mechanical switches. With passive filters, two goals can be meet simultaneously.

One that compensates for the reactive power and reaches a power factor greater than 0.9. Secondly, with a small change in the values of the inductor and the filter capacitor, the harmonics of a particular order were drowned, thereby eliminating the high-harmonic harmonics. To eliminate the rest of the harmonics, the filters (bridge to neutral) and low pass (resistance and harmonic barrier) were used. By removing the control loop to compensate for the current disturbances, we can remove the PI controller from the reference loop of the reference and DC voltages and, by adding some resistance after the shunt and DC capacitor, control the drain and charge time of the capacitor.

\section{5- Conclusion}

We managed to have a very simple but optimal control, without the need for any latent controller, complicated transformations and calculations, and, naturally, the processor to achieve a comprehensive compensation. Thus, using a hysteresis loop can achieve an acceptable and high speed compensation. If we can use this compensator at distributor stations or have a near-consumer distribution post, we will not need pll due to access to the voltage level of $20 \mathrm{kV}$ and appropriate reference if even asymmetries occur, and all disturbances will be compensated There are 2 differences and overall advantages in this type of control over other controls:1. Compensation for the moment and all in terms of using the Hysteresis band2- Controlling the charge current of the capacitor dc by capacitance and serial resistance with the capacitor, instead of using the PI controller or fuzzy control.

\section{6- References}

[1] Hassan Monsef, Behnam Tamimi,'”The Operation of dvr in Voltage Sag Mitigation Using emtp,'International Journal of Factory Automation, Robotics and Soft Computing, Issue2, April 2006 Page 102.

[2] George, Moleykutty. "Artificial Intelligence Based Three-Phase Unified Power Quality Conditioner." Journal of Computer Science 3, no. 7 (July 1, 2007): 465-477. doi:10.3844/jcssp.2007.465.477.

[3] Monteiro, L.F.C., M. Aredes, and J.A. Moor Neto. "A Control Strategy for Unified Power Quality Conditioner." 2003 IEEE International Symposium on Industrial Electronics ( Cat. No.03TH8692) (n.d.). doi:10.1109/isie.2003.1267280.

[4] Laxmi, A. Jaya, G. Tulasi Ram Das, and K. Uma Rao. "Role of PI and Fuzzy Controllers in Unified Power Quality Conditioner." Journal of Engineering and Applied Sciences 2, no. 2 (2007): 1-10.

[5] Graovac, Dušan, V. Katic, Alfred Rufer, and J. Knezevic. "Unified power quality conditioner based on current source converter topology." In EPE 2001: European Conference on Power Electronics and Applications, no. LEI-CONF-2005-065. 2001.

[6] Benysek, Grzegorz. "Experimental investigations of the UPQC arrangement." Electrical Power Quality and Utilisation. Journal 12, no. 1 (2006): 27-34.

[7] Chen, Y., L. Sanchez, K.Y. Smedley, and G. Chen. "One-Cycle Controlled Unified Power Quality Conditioner For Load Side Voltage Sag Compensation.” IEEE 36th Conference on Power Electronics Specialists, 2005. (n.d.). doi:10.1109/pesc.2005.1581636.

[8] Graovac, Dusan, Vladimir Katic, and Alfred Rufer. "Universal power quality system-an extension to universal power quality conditioner." In EPE-PEMC 2000: 9th International Conference on Power Electronics and Motion Control, no. LEI-CONF2005-051. 2000.

[9] Benachaiba, Chellali, Ottmane Abdelkhalek, Samira Dib, and Mohamed Haidas. "Optimization of parameters of the unified power quality conditioner using genetic algorithm method." Information technology and control 36, no. 2 (2007).

[10] Fujita, H., and H. Akagi. "The Unified Power Quality Conditioner: The Integration of Series- and Shunt-Active Filters." IEEE Transactions on Power Electronics 13, no. 2 (March 1998): 315-322. doi:10.1109/63.662847.

[11] Han, B., B. Bae, S. Baek, and G. Jang. "New Configuration of UPQC for Medium-Voltage Application.” IEEE Transactions on Power Delivery 21, no. 3 (July 2006): 1438-1444. doi:10.1109/tpwrd.2005.860235.

[12] Rufer, A., V. Katic, and D. Graovac. "Power Quality Compensation Using Universal Power Quality Conditioning System." IEEE Power Engineering Review 20, no. 12 (2000): 58-60. doi:10.1109/39.890381. 
[13] Zhu, Peng-Cheng, Xun Li, Yong Kang, and Jian Chen. “Analysis and Experimental Verification of a Control Scheme for Unified Power Quality Conditioner." International Journal of Energy Technology and Policy 3, no. 3 (2005): 253. doi:10.1504/ijetp.2005.007264.

[14] Jindal, Amit Kumar, Arindam Ghosh, and Avinash Joshi. “Interline Unified Power Quality Conditioner.” IEEE Transactions on Power Delivery 22, no. 1 (January 2007): 364-372. doi:10.1109/tpwrd.2006.881581.

[15] Zhu, P., X. Li, Y. Kang, and J. Chen. "Control Scheme for a Universal Power Quality Manager in a Two-Phase Synchronous Rotating Frame." IEE Proceedings - Generation, Transmission and Distribution 151, no. 5 (2004): 590. doi:10.1049/ipgtd:20040636. 